\title{
Molecular Rotors Built in Porous Materials
}

\author{
Angiolina Comotti, Silvia Bracco, Piero Sozzani*
}

Department of Materials Science, University of Milano Bicocca, Via R. Cozzi 55, 20125 Milan, Italy.

\section{CONSPECTUS.}
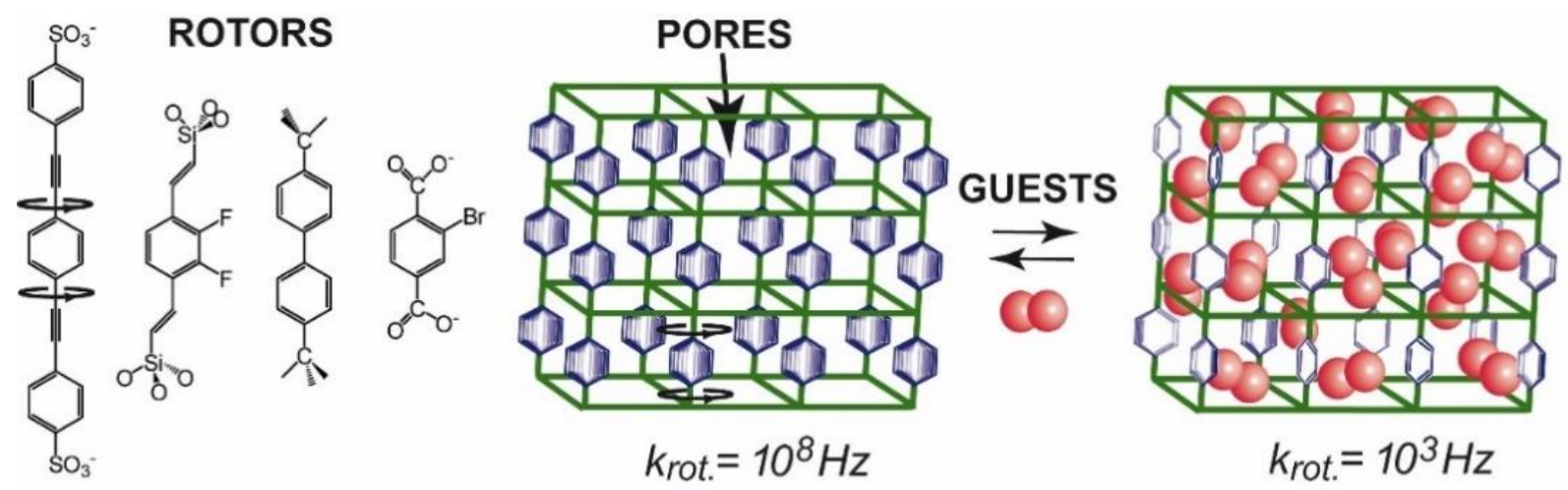

Molecules and materials can show dynamic structures in which the dominant mechanism is rotary motion. The single mobile elements are defined as 'molecular rotors' and exhibit special properties (compared to their static counterparts), being able to greatly modulate the dielectric response and form the basis for molecular motors that are designed with the idea of making molecules perform a useful mechanical function. This opens the way to challenging properties, among which is the transformation of light into useful energy. The construction of ordered rotary elements into a solid is a necessary feature for such design, because it enables the alignment of rotors and the fine tuning of their steric and dipolar interactions. Crystal surfaces or bulk crystals are the most suitable to adapt rotors in 2D or 3D arrangements and engineer juxtaposition of the rotors in an ordered way. Nevertheless, it is only in recent times that materials showing porosity and remarkably low density have undergone tremendous development. The characteristics of large free-volume combine well with the virtually unhindered motion of the molecular rotors built into their structure. Indeed, the molecular rotors are used as struts in porous covalent and supramolecular architectures, spanning both hybrid and fully-organic materials. The modularity of the approach renders possible a variety of rotor geometrical arrangements in both robust frameworks stable up to $850 \mathrm{~K}$ and self-assembled molecular materials. A nanosecond (fast dynamics) motional regime can be achieved at temperatures lower than $240 \mathrm{~K}$, enabling rotor arrays operating in the solid state even at low temperatures. Furthermore, in nanoporous materials, molecular rotors can interact with the diffusing-in chemical species, be they liquids, vapors, or gases. Through this chemical intervention rotor speed can be modulated at will, enabling a new generation of rotor-containing materials sensitive to guests. On the other hand, an applied electric field can be the stimulus for chemical release from porous materials. The effort needed to obtain strong dipoles, which are non-centrosymmetrically mounted onto rotors and which do not hamper rotational motion, is a further aspect of this research activity. This has led to challenging materials that are promptly responsive to an applied electric field, altering the ferroelectric or anti-ferroelectric ground state by fast dipole re-orientation when subjected to electric polarization. Thus, materials showing switchable dielectric properties in response to applied electric fields have been fabricated.

\section{INTRODUCTION}

Motional phenomena occurring in the solid state have been frequently envisaged as a way to conjugate the dimensional stability and mechanical properties of solid materials with the flexibility and dynamics of fluids. Descriptions have already been given of several solid materials, endowed with intrinsic dynamics, wherein some components of the structure possess enough freedom to undergo fast, thermally activated, motion. ${ }^{1-3}$ In addition to the example of soft materials, such as cross-linked polymers above their glass transition, even shape-persistent structures, such as crystals, can show remarkable dynamics in their various components. Indeed, hard matter can contain mobile elements and flexible molecular moieties, which can be designed to induce new and interesting properties. The efforts of engineering these molecular elements, in both a rational fashion and possibly in a concerted way, has enabled the 
construction of molecular motors and molecular devices. ${ }^{4-6}$ In this context, molecular elements, which perform simple motion trajectories and, in particular, rotary motion (molecular rotors) play a major role in performing special functions: for instances dipoles mounted on molecular rotors may lead to switchable organic ferroelectric materials. Actually, tunable dielectrics are obtained if rotors are ordered in 2D or 3D arrays. Concerted rotor dynamics of dipoles in such regular environments can generate slow wave propagation in the radiofrequency regime, similar to acoustic waves, and can be used to built radio-frequency filters. Therefore, dipole-dipole interactions in anisotropic arrangements such as ordered solids and crystalline-like aggregation states open challenging perspectives in signal processing. In other applications, the switching of molecular rotation takes place in association with a change of spin state, birefringence or electric conductivity. ${ }^{7-10}$

Molecular dynamics in solids is associated with the presence of available space, sometimes called free-volume, which allows motion to occur in a low-density material. This empty room typically accounts for a few percentiles of the total volume, but it allows to achieve enough freedom for the dynamical moieties. This principle is well-documented in amorphous materials such as polymers above their glass transition and hexagonal rotator phases of linear alkanes. ${ }^{3,11-12}$ Thus, low-density is a prerequisite for the design of mobile elements in solids and this principle has been exploited in a variety of materials, the stimulus being to obtain new molecular mobility benchmarks. ${ }^{2}$

A major breakthrough was achieved when a remarkable amount of free-volume, and consequently of molecular freedom, was provided by permanently porous solids. Indeed, some materials were engineered to generate low-density architectures in which a large excess room was tailored to form galleries or channels, exposing mobile elements to an empty space. Thus, the highly porous materials of the last generation constitute a playground for enhancing dynamics in the solid state. ${ }^{13-15}$ Such challenging systems can 'breath' through the pores by guest adsorption/desorption and the entire population of rotors interacts with molecular species diffusing in the material. Such direct interaction has been exploited to regulate rotor speed at will, thanks to the specific nature of the molecules diffused in the nanopores, building up a pervasive and tunable interface.

Furthermore, porous solids can integrate into their 3D structures a second component that can behave as a dynamic nanophase (sub-structure). This is the case of guest molecular rotors assembled in an orderly fashion in hostguest binary structures. ${ }^{3}$ This versatile method allows the realization of guest rotary motion without the formation of covalent bonds between the rotary axis and the framework, although the control over the motional trajectories is sometimes problematic.

Collectively, the combination of two properties in a same material, i.e. fast rotors and the existence of structural pores which offer large accessible porosity, feeds the field of molecular rotors and motors with exuberant perspectives still to be explored. They include coherent dynamics in oriented systems and the opportunity of intervening through the accessible pores on performing solid-state reactions, which enable the construction of sophisticated machinery. The anisotropic re-orientation and rotor alignment allows for macroscopic manipulation of vectorial properties, such as dielectric phenomena.

Porous materials containing rotors have been realized by self-assembly of suitable organic or hybrid building blocks following a few strategies, which involve covalent bonds, metal-organic or supramolecular interactions, that sustain their architectures. They will be described in the following, starting from the prominent group of hybrid materials and metal organic frameworks MOFs and later shifting to fully organic covalent and supramolecular networks.

\section{ROTORS IN HYBRID MATERIALS}

\subsection{Mesoporous Organo-Silicas}

Periodic Mesoporous Organosilicas (PMOs) consist of fully covalent architectures, and conjugate the thermal robusteness of siloxane bonds, typical of silica, with the chemical stability of carbon-carbon organic bonds. ${ }^{16}$ They generally belong to the family of mesoporous materials since they exhibit cylindrical pores of more than $2 \mathrm{~nm}$, obtained by condensation of organic-inorganic building blocks on the scaffold of templating micelles. The reaction of bis-trialkoxyorganosilanes leads to the formation of three siloxane bonds at each molecular end, thus composing a cross-linked porous structure constituted by a large part of organic, especially aromatic, matter (Figure 1). The self-assembly process not only allows the generation of a single network of alternated organic and inorganic nanolayers, but also, under suitable conditions, the precise organization of periodically aligned monomer units in the framework.

XRD clearly indicates two hierarchical levels of order, both on the molecular scale and the nanometers scale. While the latter derives from the hexagonal arrangement of the channel-like pores, the molecular order demonstrates that the organic linkers are set in a favorable manner to engineer molecular rotors in ordered arrays. The molecular order is compatible with even rather elongated organic linkers, for instance aromatic units substituted in para positions or diphenyls, which exhibit the pivotal bonds lying on a rotational axis. Other favorable features for the construction of rotors is the distance at which the rotational axes are set, in fact, the siloxane bonds act as spacers to keep the rotors apart, one from the other.

Molecular rotors in mesoporous organosilicas were first described in 2008 in diphenylsilica which, at that time, was the prototype example of rotors in porous materials (Figure 2). ${ }^{17}$ The discovery was based on the collection of both ${ }^{1} \mathrm{H}$ and ${ }^{13} \mathrm{C}$ spin-lattice NMR relaxation times $\left(\mathrm{T}_{1}\right)$ at variable temperature, these being most sensitive to motional phenomena over a wide temperature range $10^{3}-10^{8} \mathrm{~Hz}$. The maximum relaxation rate occurs when thermal agitation frequencies match the observation frequency, according to the formula $\tau \times \omega \cong 1$. The data can be fitted by the KuboTomita equation, which describes the modulation of relaxation by carbon-hydrogen vector reorientation. ${ }^{18}{ }^{13} \mathrm{C} \mathrm{T}_{1}$ relaxation times recorded at $75 \mathrm{MHz}$ showed a maximum at $310 \mathrm{~K}$, indicating that at this temperature the rotors respond in the $10^{7} \mathrm{~Hz}$ range. ${ }^{1} \mathrm{H} \mathrm{T}_{1}$ relaxation times at 300 and $30 \mathrm{MHz}$ confirm that the sample response falls in this 
motional regime with interconversion energy barriers as low as $6.4 \mathrm{kcal} / \mathrm{mol}$.
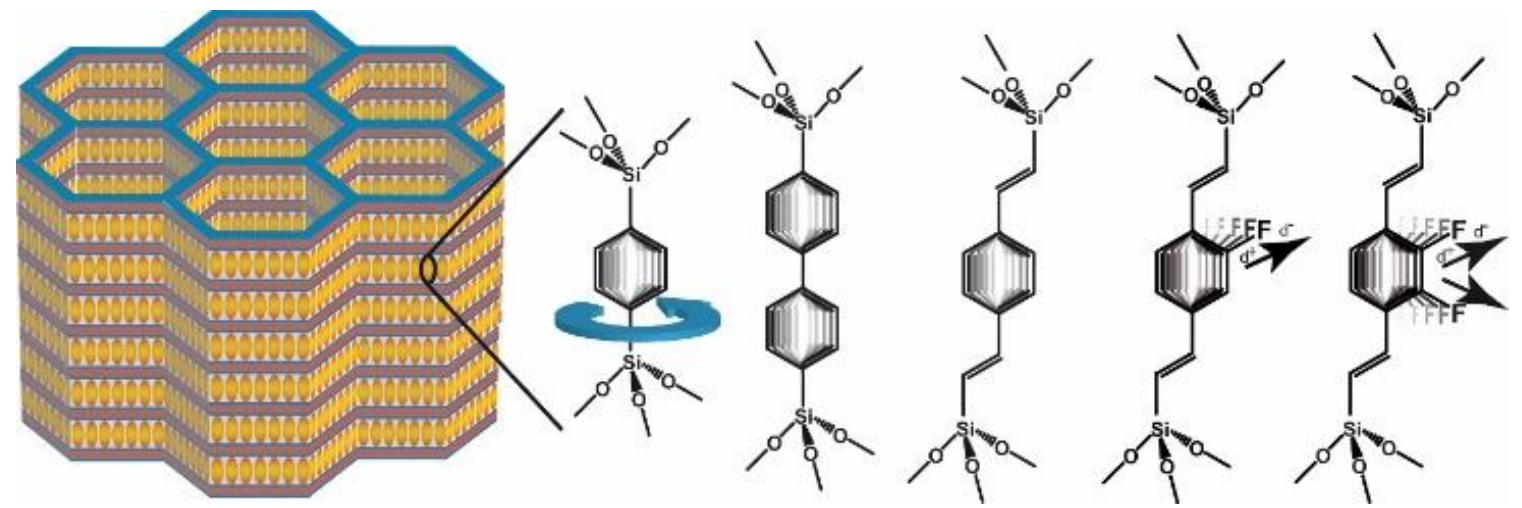

Figure 1. Representation of periodic mesoporous organosilicas and the various linkers.

a)

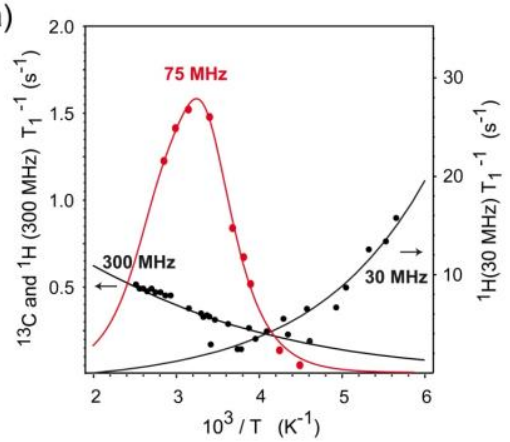

b)

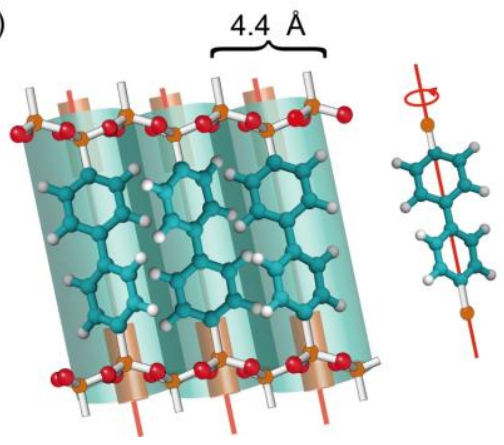

Figure 2. a) Temperature-dependent ${ }^{1} \mathrm{H}$ relaxation rates at 30 and $300 \mathrm{MHz}$ and ${ }^{13} \mathrm{C}$ relaxation rates at $75 \mathrm{MHz}$ (for $\mathrm{CH}$ carbon) for mesoporous diphenylsilica. b) Schematic picture of rod-like diphenyl rotors vertically aligned along the channelaxis.

This result stimulated the study of other p-phenylene rotor-containing periodically ordered PMOs. A direct proof of the motional mechanism can only be derived by an unvaluable method such as ${ }^{2} \mathrm{H}$ spin-echo NMR. ${ }^{19}$ It requires the preparation of deuterated samples with C-D bonds in specific positions on the rotary part. If reorientational frequencies $\mathrm{k}$ are slower than $10^{3} \mathrm{~Hz}$ as occurs in rigid structures, the C-D bond cannot move significantly, thus a typical Pakepattern spectrum with a singularity spacing of $d=125-136$ $\mathrm{kHz}$ for aliphatic or aromatic carbons is detected. Faster motional regimes produce signal averaging and articulated profiles which can be simulated by anisotropic motional models. Deuterated para-phenylenesilica showed ${ }^{2} \mathrm{H}$ NMR differentiated patterns as a function of temperature: starting from the 'static' (Pake pattern) spectrum detected at low temperature, a dramatic evolution of the spectrum profile occurs on increasing temperature, the signal intensity increasing towards the central part of the spectrum, which is an indication of motional averaging (Figure 3). ${ }^{20}$ Intermediate motional regimes give rise to profiles with multiple singularities and a complex shape. The simulation of the patterns according to the trajectories of the C-D bonds yielded rotor speed at each particular temperature. The spectra show singularities separated by $33.4 \mathrm{~Hz}$ with the shape of a restricted Pake spectrum of $d / 4$, which can be interpreted by two-site $180^{\circ}$ flip reorientation of the phenyl rings about their axes. The rate of the rotors reaches values as high as $10^{8} \mathrm{~Hz}$ at room temperature, but the thermal robustness of the carbon-carbon, carbon-silicon and silicon-oxygen covalent bonds allowed observations of dynamic moieties at considerably high temperature with substantial mechanism stability. From the Arrhenius plot we can deduce a remarkably low activation energy of $6.2 \mathrm{kcal} / \mathrm{mol}$ and the high frequency limit of $2 \times 10^{12} \mathrm{~Hz}$, in agreement with the small pphenylene rotational inertial mass. ${ }^{2,21}$

The direct NMR demonstration of p-phenylene-organosilica nanochannel accessibility, detected by diffused-in hyperpolarized Xe-gas, ${ }^{22}$ prompted us to perform a totally new experiment: actually, the open-porosity of the material offered the perspective of regulating rotary motion by guest stimuli. Indeed, when p-phenylenesilica channels were filled with guest molecules such as octadecyltrimethylammonium bromide, a drastic reduction of the rotational rates by three orders of magnitude was observed and the activation energy increased up to $10 \mathrm{kcal} / \mathrm{mol}$. The energy barriers increment between guest-filled and empty channels thus resulted in $4 \mathrm{kcal} / \mathrm{mol}$, such measurement allowed to estimate, in an unusual way, the weak intermolecular interactions occurring at the host-guest extended interfaces. Modulation of rotor dynamics was obtained by a variety of guests, including $\mathrm{H}_{2} \mathrm{O}$ (not presented), showing for the first time the possibility to tune the rotor speed in porous materials (Figure 4).

Periodic order has been maintained in mesoporous organosilicas constructed with a major amount of organic component and rotor-containing elongated linkers. In the particular case of divinyl-p-phenylenesilica the rotor lies between two carbon-carbon double bonds that set the rotors at a distance from the inorganic layers (Figure 5). 
A)

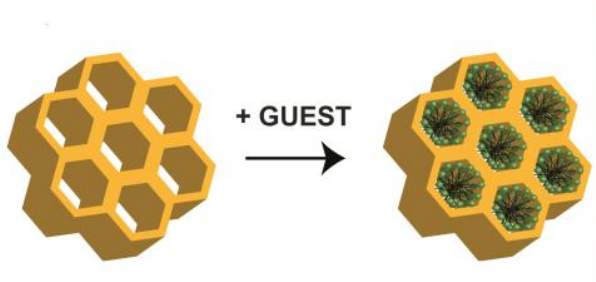

empty pores

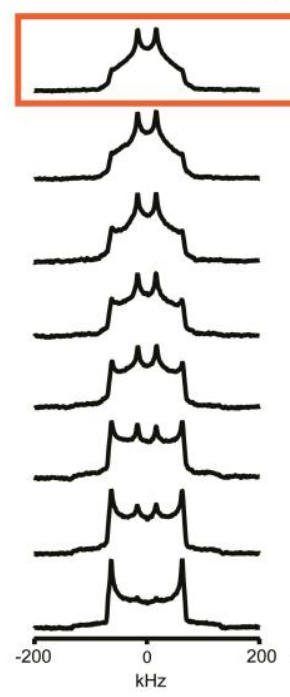

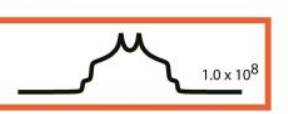
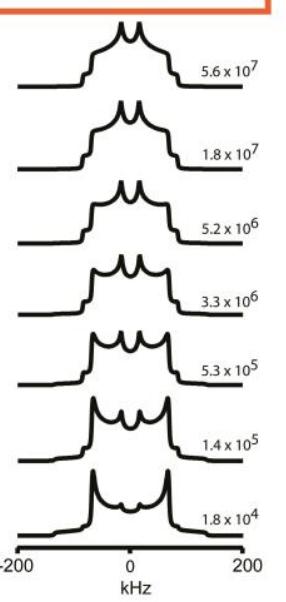

B)
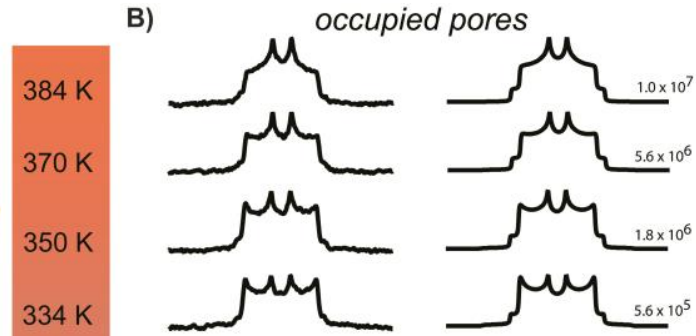

$318 \mathrm{~K}$

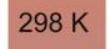

$282 \mathrm{~K}$

$265 \mathrm{~K}$

$250 \mathrm{~K}$

$235 \mathrm{~K}$

$215 \mathrm{~K}$

$196 \mathrm{~K}$

$164 \mathrm{~K}$
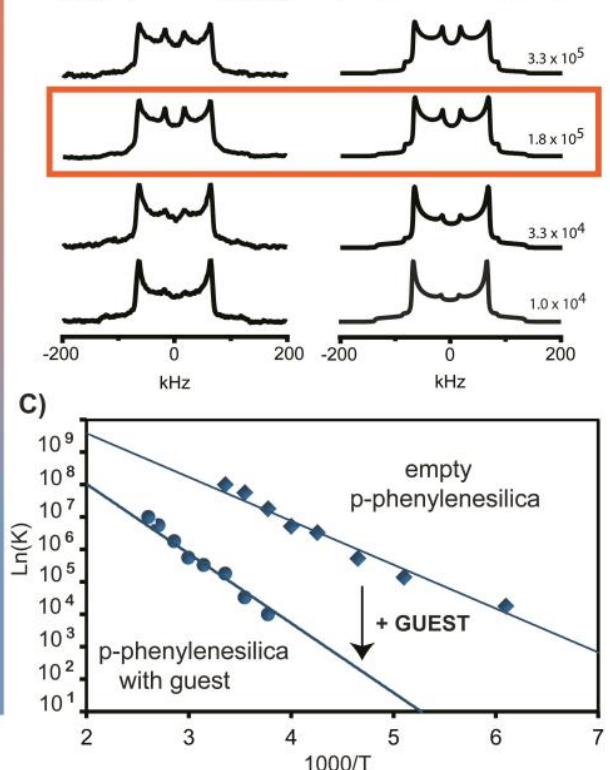

Figure 3. ${ }^{2} \mathrm{H}-\mathrm{NMR}$ spectra of $\mathrm{d}_{4}$-p-phenylenesilica compared to the simulated spectra for A) empty nanochannels and B) guest-filled nanochannels. Exchange rates $\mathrm{k}(\mathrm{Hz})$ are reported. C) Arrhenius plot of the two compounds.

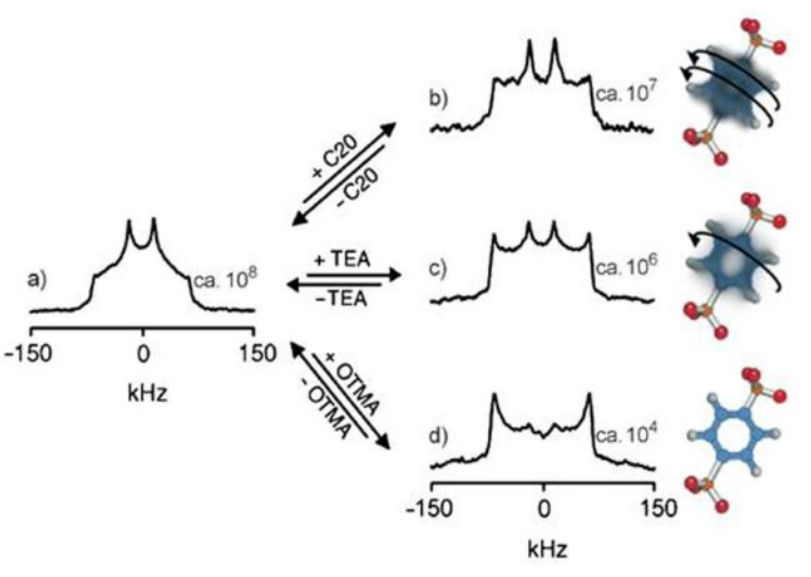

Figure 4. ${ }^{2} \mathrm{H}$ NMR spectra of mesoporous p-phenylenesilica with b) C20, c) tetraethylammonium and d) octadecylammonium compared to the empty matrix (a). The exchange rates (Hz) are reported.

This geometrical arrangement results in the organic elements gaining a great degree of freedom: the compound deuterated on the phenyl ring shows a rapid C-D bond reorientation in the $\mathrm{MHz}$ regime at room temperature, but, more interestingly, a transition to much faster motion about room temperature takes place. ${ }^{23}$ In fact, an abrupt rotational dynamics change, clearly non-Arrhenius behavior, speed up the motional regime to become extremely fast. At higher temperature, there occur much wider librations manifested by the apparent weakening of the spectral shoulders. The transition, involving a gain of motional freedom, is associated with an increase in heat capacity, as observed in differential scanning calorimetry trace showing the appearance of a glass transition (Tg).

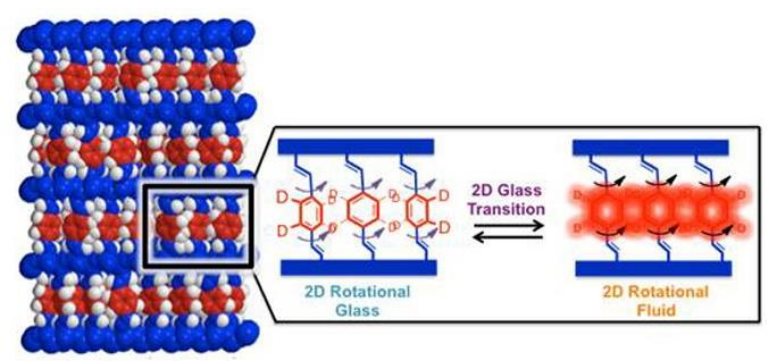

Figure 5. Schematic representation of the divinyl-p-phenylenesilica wall and its 2D-glass transition.

\subsection{Rotors Connected By Metal Nodes In Metal-Or- ganic Frameworks}

MOFs or Porous Coordination Polymers (PCPs) is one of the most attractive area in the current research as they hold 
promises for gas capture, molecular separation, catalysis, polymerization and optical or electronic properties. The term 'dynamics' in MOF structures regards intrinsic framework flexibility based on switching between two differently porous architectures, or is used to indicate the insertion of mobile moieties in the fixed reference system of the framework. In the latter case, rotors have been found in a few families of MOFs. In cadmium-based PCP containing pyrazine linkers pivoted on the metal node, the specific configuration ensures an extremely low barrier (approx. $2 \mathrm{kcal} / \mathrm{mol}$ ) for the 4-site rotation about the metal-organic bonds placed on the same axis (Figure 6). ${ }^{24}$ Nevertheless, the low attempt frequency of $10^{6} \mathrm{~Hz}$ limits the spinning speed to moderate motional regimes of $10^{5} \mathrm{~Hz}$ at room temperature. Furthermore, MOFs dicarboxylate aromatic ligands, like terephthalate, display the $\mathrm{Csp}_{2}-\mathrm{Csp}_{2}$ pivotal bonds with a partial conjugation to the aromatic electronic system and a high activation energy for rotation, 10 to $15 \mathrm{kcal} / \mathrm{mol}$, rendering the flipping motion sufficiently fast only at high temperatures $(450 \mathrm{~K})^{2,25,26}$

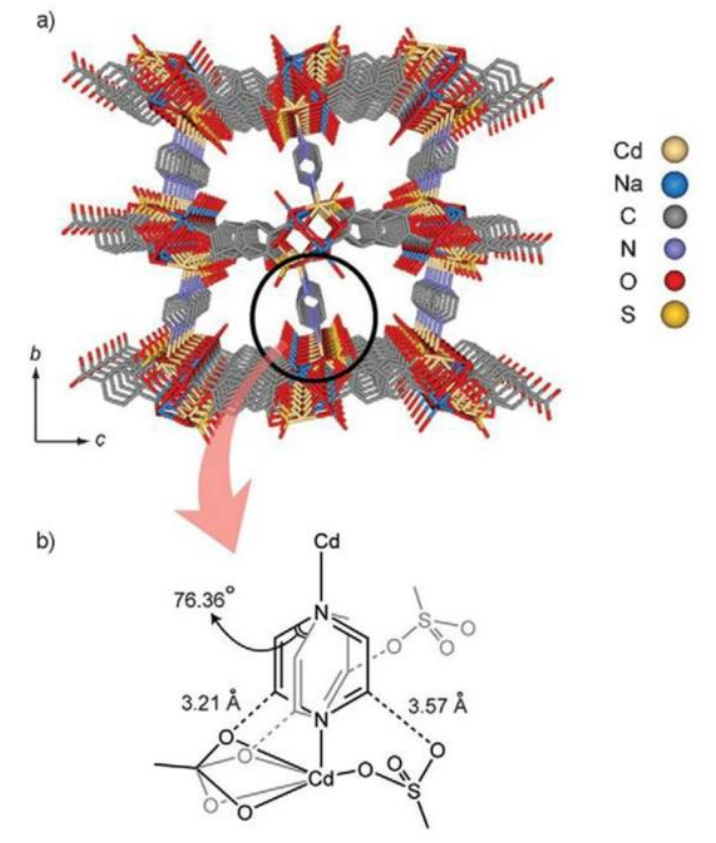

Figure 6. Crystal structure of the Cd-based MOF: a) the pillared layer structure. b) Pyrazine rotor.

The choice of para-phenylene as the basic rotary unit was the most attractive for its low molecular mass: it was discovered that the additional organic functions on the phenylene ring are not necessarily a burden that hampers rotation, but they may be beneficial to the flip motion since the lateral substituent destabilizes the ground state. Moreover, the introduction of functionalities carrying dipoles into rotators brought about additional benefits. In the case of IRMOF-2, the linker is the Br-terephthalate group with the $\mathrm{Zn}$ metal node. Dielectric response was observed in the audio frequency regime, and found to follow a hopping model of molecular dipole relaxation, with a single energy barrier of $7.3 \mathrm{kcal} \mathrm{mol}^{-1}$. $^{27}$ The behavior of IRMOF-3, which contains the aminic linker, showed by ${ }^{1} \mathrm{H}$ NMR relaxation, allowed a decrease in the energy barrier down to $5 \mathrm{kcal} / \mathrm{mol}$ and the infinite-temperature frequency decreased by six orders of magnitude $\left(10^{7} \mathrm{~Hz} \text { vs } 10^{13} \mathrm{~Hz}\right)^{28}$

The rather constrained local environment for the phenylene rings in $1 \mathrm{D}$ pore systems of MIL-47(V) and MIL$53(\mathrm{Cr})$, compared to a 3D system such as MOF-5, was reflected in the variable temperature ${ }^{2} \mathrm{H}$ NMR experiments. ${ }^{25}$ Although the spectra are becoming rather distorted by the effect of 8 paramagnetic centers in close proximity, it has been possible to determine the activation energy of 10-11 $\mathrm{Kcal} / \mathrm{mol}$.

\section{ROTORS IN MOLECULAR CRYSTALS}

\subsection{Molecular Porous Materials}

Porous Molecular Materials offer stimulating perspectives in building crystalline architectures by exploiting the versatility of the organic synthesis. Only a few were described until recent years. ${ }^{29-32}$ This is likely due to the objective difficulties in preventing the tendency of molecules to aggregate into a close-packed crystal, thus cancelling any significant porosity. In fact, the absence of intermolecular interactions as strong and directional as covalent bonds, is manifested by the tendency to structural collapse. Indeed, until recent years, the principle by which any molecular system could reach its maximum close packing was considered inviolable. However, structural stability can be notably increased if molecular rigidity and appropriate shape factors of the molecules reduce favorable packing. ${ }^{33,34}$ Porous architectures can be induced by host-guest formation followed by mild guest removal. This process results in crystals with permanent pores, which are generally accessible from the gas phase. Hydrogen-bonding and Coulombian interactions may contribute to stabilize low-density frameworks. Indeed, rod-like molecules containing aromatic rings are most suitable to engineer rotors appended in their core, like a wheel mounted on an axel. Rotors based on p-phenylene moieites and lying between two triple carboncarbon bonds produce a rotational energy barrier lower than $1 \mathrm{kcal} / \mathrm{mol}^{35}$ Triple bonds extend the length of the rotational axis while a further extension of the molecular axis can be achieved by additional p-phenylene units at the end of molecule as depicted in Figure 7. The rod-like molecules bearing sulfonate groups at their ends self-assemble with alkylammonium cations, forming a porous architecture held together by charged-assisted hydrogen bonds.

Spin-lattice relaxation times of the carbon nuclei thoroughly describe the motional behavior of each molecular fragment: the two peripheral p-phenylene rings show, in the examined temperature range, relatively long relaxation times in the order of 20-40 s, indicating that they are far from being interested by rotation. On the contrary, the central ring finds a most efficient relaxation with $\mathrm{T}_{1}$ relaxation times as short as 0.25 seconds, which, at the applied magnetic field of 7 Tesla, is diagnostic of rotary motions with correlation times in the nanosecond regime. Contrarily, carbon nuclei lying on the molecular axes are substantially unaffected by motion, in fact they display considerably long relaxation times. This observations are fully consistent with spin-echo deuterium NMR spectra at variable temperature which depict C-D bonds of the labelled central ring rapidly reorienting about the rotation axis. This mechanism 
persists in a range extended down to very low temperatures, showing extremely fast spinning speed such as $10^{7} \mathrm{~Hz}$ even in the 200-240 K range.

Moreover, the central ring is fully exposed to adjacent channels and may be sensitive to gases flowing-in. The material was exposed to $\mathrm{I}_{2}$ vapors and, already at very low iodine pressure, the rotor dynamics was affected (Figure 8). Actually, the energy barrier for rotation was drastically increased up to $10.5 \mathrm{kcal} / \mathrm{mol}$. The rotor dynamics could be switched off and on by $\mathrm{I}_{2}$ absorption/desorption, showing remarkable response of material dynamics to the interaction with volatile species, suggesting the use of molecular crystals in sensing and pollutant management.

a)

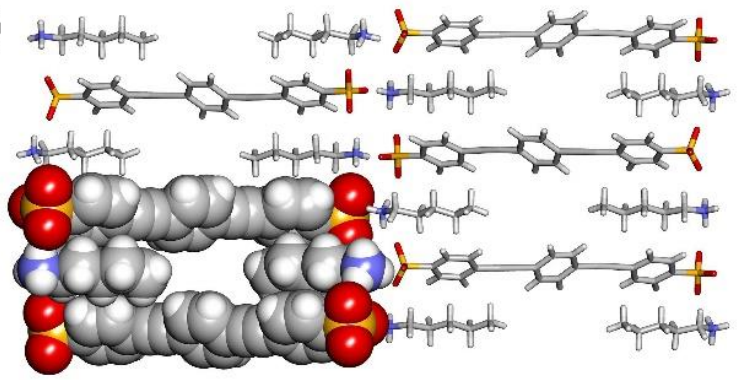

b)
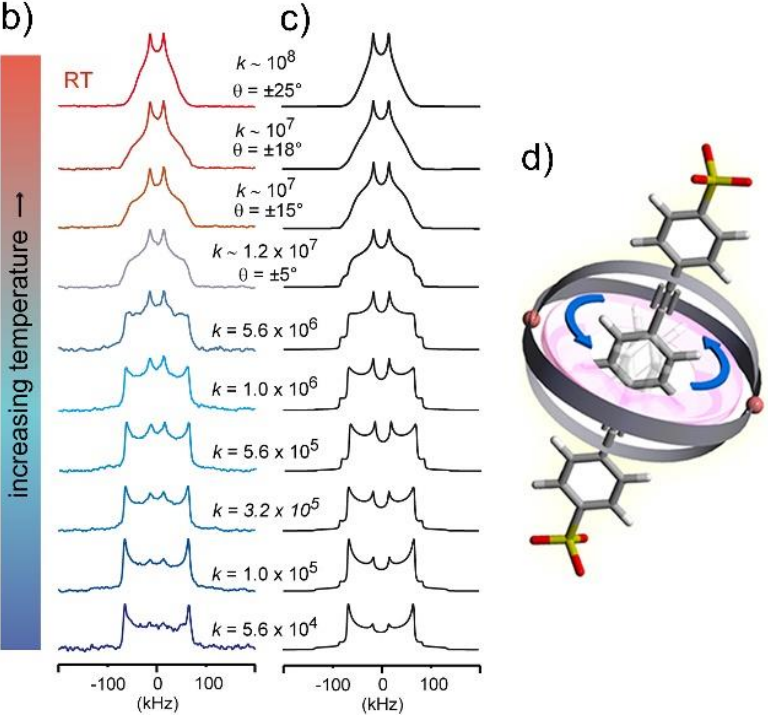

Figure 7. a) Crystal structure of porous compound. b) Experimental and c) simulated ${ }^{2} \mathrm{H}-\mathrm{NMR}$ spectra as function of temperature. The energy barrier of rotor speed accounted for 4.7 $\mathrm{kcal} / \mathrm{mol}$. d) 4,4-bis(sulfophenylethynyl)benzene linker showing the central p-phenylene rotor.
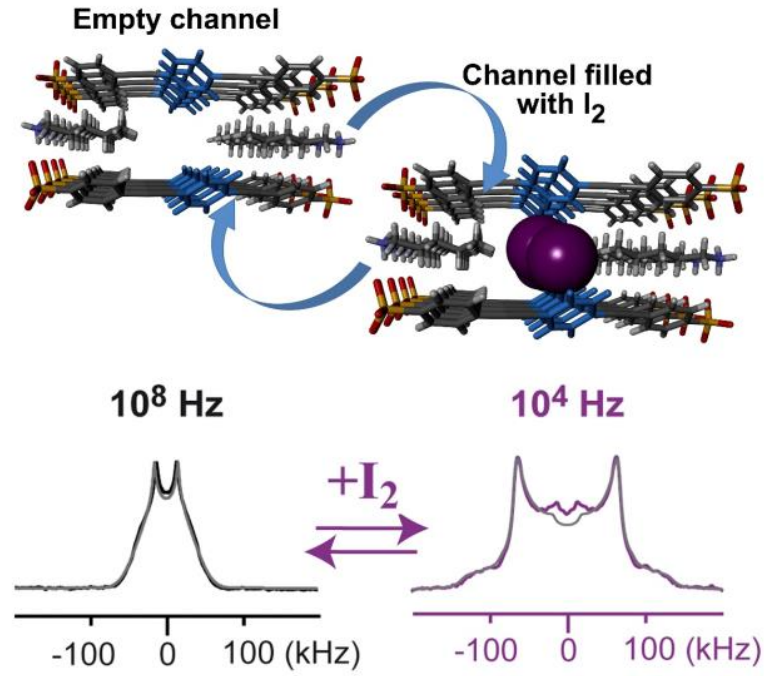

Figure 8. Structure and ${ }^{2} \mathrm{H}-\mathrm{NMR}$ spectra of empty (left) and iodine-loaded (right) compound. The exchange rates are reported.

\subsection{Rotary Motion of Guests In Host-Guest Sys- tems}

The vast majority of guest molecules, when included in solid hosts, find a way to gain at least some degrees of motion. The host dictates the symmetry and the anisotropic shape of the cavities and, in response, the included guests increase their motion, 'dynamically' occupying the overall space available by multiple and dynamically-explored conformations. Large oscillations or non-axially symmetric reorientations are commonly observed in inclusion compounds but they do not generate single-axis molecular rotors. In fact, many of host-guest systems are clathrates, wherein rotary motion cannot be systematically engineered.

Porous materials provide a suitable predetermined room for guests to be included, as shown by the versatility of such systems in controlling the anisotropic motion of numerous diverse guests. Elongated and polymer guests find their perfect complement in host crystalline nanochannels: this prerogative was recognized several years ago and gave rise to fast spinning of the guest chains about their main axes. Long segments of the polymer chains underwent diffusional rotation even for high molecular mass polymers. Hence, low molecular mass hydrocarbon chains displayed rotational behavior in quasi-cylindrical crystalline channels. ${ }^{36-42}$ In fact, tight channels promote fast dynamics, not restrict motion, compared to much larger and comfortable channels wherein flexible polymer chains relax to intricate conformations that may convert into each other in a concerted and complex way, thus gaining space occupation without extended chain-segment spinning. This indicates that complementary host-to-guest conditions play a key role in promoting rotary motion and, in particular, a circular crosssection is compatible with the rotational motion. The spinning of extended polymer chains in nanochannels mimics the rotator phases observed at high pressure/temperature 
in bulk polymers and hydrocarbons, and enabled to elucidate the motional state of polymers in the bulk phase, which is of importance to understand heat exchange and frictional behavior. ${ }^{11}$

An extension of the above concepts lead to the formation of channel inclusion compounds with suitable guests. ${ }^{43,44}$ Such guests could be engineered with end segments capping the rotary moiety, which is appended to the central part of the elongated molecule: thiophene oligomers showed ultrafast mobility when covalently connected to two alkoxy chain-ends. Ultra-fast motion regimes were achieved, as MAS NMR and laser assisted Fourier Transform ESR put in evidence.

The inclusion-compound approach was exploited in an innovative way to produce molecular rotors anchored on a crystal surface. The guest molecules were constructed with specialized moieties, each of them designed for a different purpose. ${ }^{45}$ Typically, they are individually constituted by rotary heads (rotators), bulky stoppers (which prevent the rotators from entering the channels) and long hydrocarbon tails, which fit perfectly in the channels and are rooted in the porous crystal by multiple weak interactions (Figure 9). The channel geometry ensures that the rotators protrude from the crystal surface and are set at a distance one from the other by the hexagonal arrangement of the host lattice. The host geometry poses the guest molecules in register and guarantees an ordered distribution on the surface, promoting motion of the polar heads that are free to reorient.

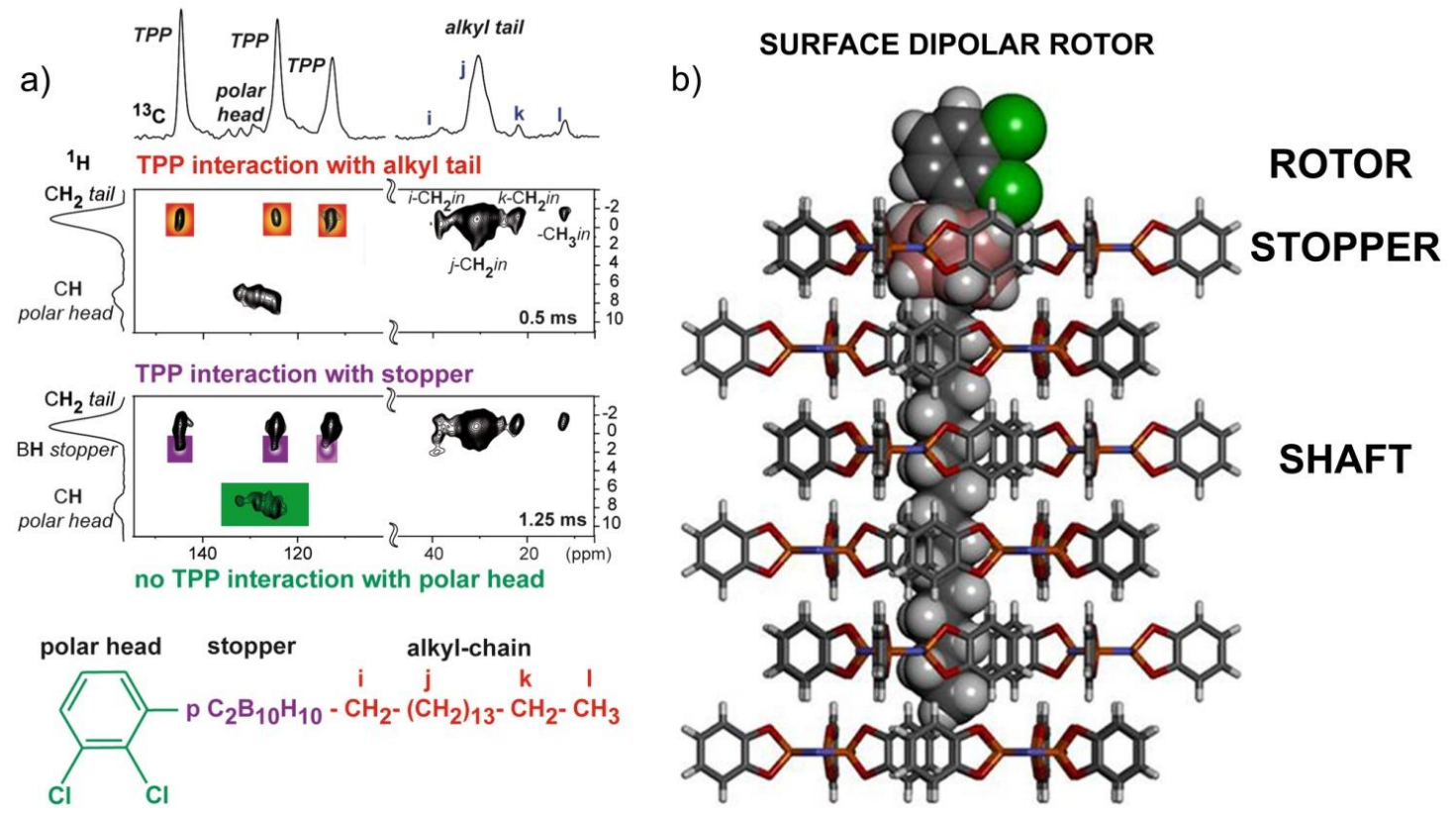

Figure 9. a) 2D ${ }^{1} \mathrm{H}-13 \mathrm{C}$ HETCOR NMR spectra of surface inclusion compound at distinct contact-times. b) Dipolar-rotor-bearing molecules inserted into tris-o-phenylenedioxycyclotriphosphazene (TPP) nanochannels.

The juxtaposition of rotor molecules has been established unconventionally by 2D MAS NMR spectra, showing a double correlation with ${ }^{13} \mathrm{C}$ and ${ }^{1} \mathrm{H}$ nuclei. The necessary condition to collect a 2D signal is the close-space proximity; independently of the covalent links, between the interacting nuclei. Therefore, the alkyl tails of the guest inserted in the

A number of publications followed the above seminal papers, giving rise to further examples of applications of TPP for the inclusion-compound approach and porous-host/rotary-guest materials engineered to realize molecular rotors in solids. A few interesting examples of spinning guests showed energy barriers favorable for rotation. ${ }^{46}$

\section{ROTORS IN POROUS ORGANIC FRAMEWORKS}

A recent and stimulating research subject is that of porous covalent frameworks, which exhibit surface areas as large as 4000-7000 $\mathrm{m}^{2} / \mathrm{g}$ and robust structures stable up to $450{ }^{\circ} \mathrm{C}$. They are mostly constituted by rigid rod-like organic struts and display galleries of about $15 \AA$, thus belonging to the category of microporous materials. The first matrix must produce host-guest strong correlation signals, while the signals for the stopper at the end of the matrix channels appear under more severe recording conditions. Importantly, the absence of correlation signals between the rotator and the host prove that the rotating heads are far from the bulk crystal.

and up-to-date example of molecular rotors in porous organic frameworks was described in the case of so-called Porous Aromatic frameworks (PAFs). ${ }^{15}$ They are substantially constituted by aromatic rings anchored covalently onto tetrahedral nodes, which can be carbon, silicon or germanium (PAF-1, PAF-3, PAF-4, respectively). The full exposure of the struts towards empty cavities provides them with a tremendous freedom, limited only by the torsion barrier at the pivotal bonds and the exposure to species visiting the galleries through the open and light architecture. Given the above premises, porous organic frameworks are perhaps the most promising and versatile materials in this field. In a first example (PAF-3), the rotor speed has been measured to be $10^{6} \mathrm{~Hz}$ already in a cold environment below 200K (Figure 10). This value is obtained by spin-echo deuterium 
NMR: the line-powder pattern fits perfectly a fast 2-site $180^{\circ}$ flip reorientation of the p-phenylene units. ${ }^{47}$ Furthermore, the abovementioned robustness of the scaffold, which is sustained by a barely deformable 3D-network allowed to study the rotor dynamics at far higher temperature than in any other organic system. This provided a unique opportunity to detect motional mechanisms under such extremely severe conditions. Actually, the way phenylene rings rotate changes appreciably in the highest temperature range. The experimental profile displays a narrower line truncated by a flat terrace on the top. This peculiar profile corresponds to a calculated spectrum consistent with a large displacement (plus/minus $37^{\circ}$ ) away from the $0^{\circ}$ and $180^{\circ}$ arrangements, where the limit positions are much more frequently explored than the intermediate ones

a)

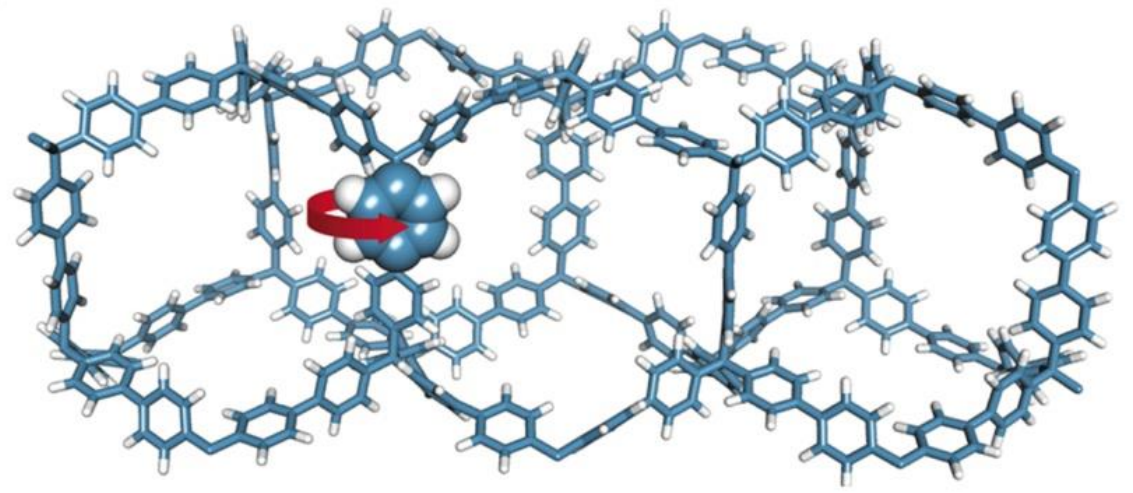

b)
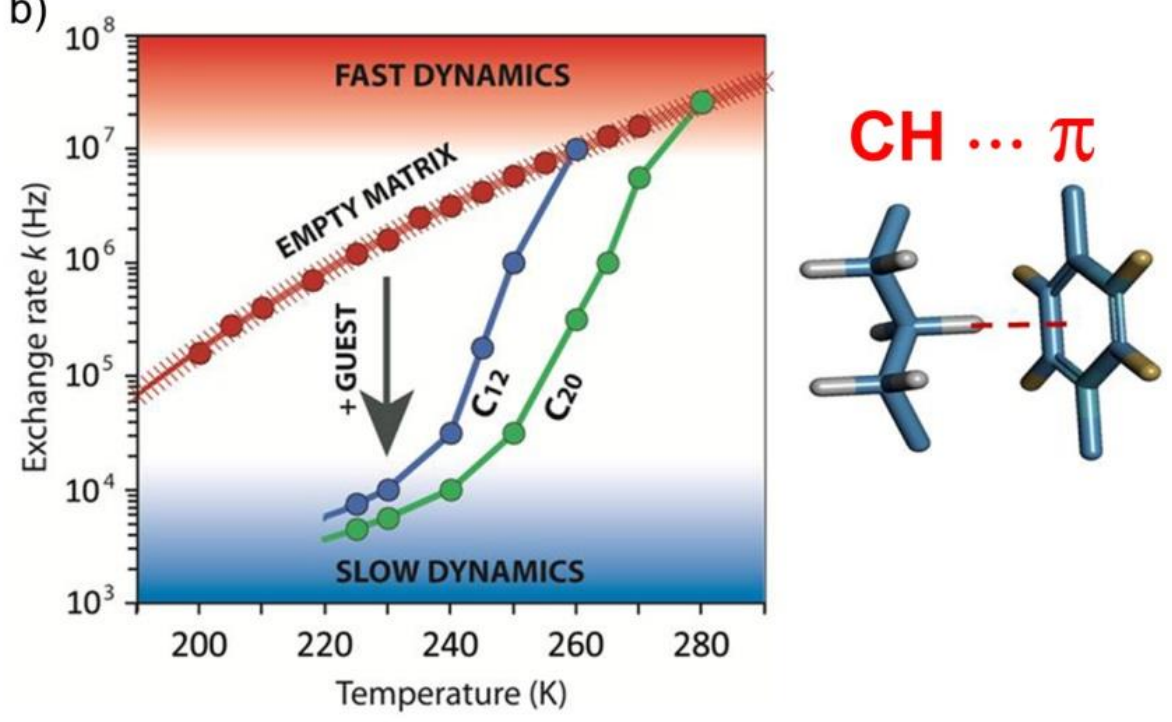

like in a pendulum model. Thus, 4 sites are populated during the rotary trajectory with extremely low interconversion barrier, and free-rotation was then approached at $550 \mathrm{~K}$. Such fast-spinning behavior was slowed down immediately after iodine vapor diffusion to the porous material. This experiment proved the high porosity by an unconventional spectroscopic detection and that the molecular entities of the material can exist in two distinct, dynamic and static, forms. The effect by which dynamics can be regained reversibly was achieved on command after a short vacuum application.

\section{C)}

LARGE LIBRATION

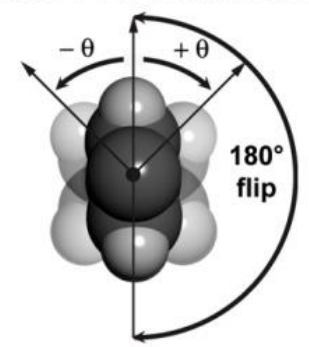

d)

d)

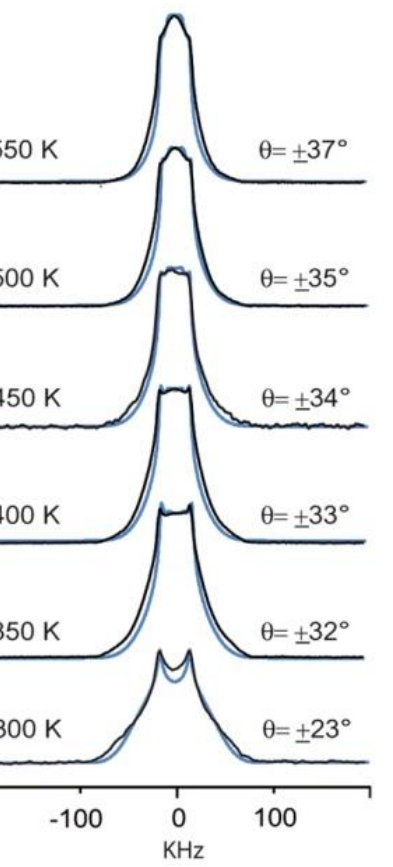

Figure 10. a) Structure of PAFs. b) Rotational rates of empty, C12- and C20-loaded d4-PAF3 vs temperature. The energy-barrier of rotor-speed in empty $\mathrm{d}_{4}$-PAF-3 accounted for $4.4 \mathrm{kcal} / \mathrm{mol}$. c) Large-amplitude p-phenylene librations occurring at high temperature along with ${ }^{2} \mathrm{H}$ NMR spectra of $\mathrm{d}_{4}-\mathrm{PAF}-3(\mathrm{~d})$.

$\mathrm{N}$-alkanes such as $\mathrm{C} 12$ and $\mathrm{C} 20$ diffused-in the nanochannels from the melt phase also act as brakes which hamper the p-phenylene rotation at sufficiently low temperature. This is due to the $\mathrm{CH} \cdots \pi$ interactions installed between the guest and the molecular rotors in the host. In fact, the energy barrier is increased in the amount of $2-3 \mathrm{kcal} / \mathrm{mol}$. On increasing temperature the rotors undergo a motional transition and switch back to regimes as fast as in the pure matrix, because the energy of thermal agitation competes with the weak $\mathrm{CH} \cdots \pi$ interactions between the two components.

\section{DIPOLAR ROTORS IN FLUORINATED MESOPOROUS MATERIALS}


The ultimate goal of the field of rotors in porous materials is the realization of rotating dipoles by the insertion of polar groups on the molecular rotor. It is not sufficient that the dipole distribution is non centrosymmmetric but it is necessary that dipole oscillations would produce the molecular dipole displacement. Therefore, the principles that enable a rotor to behave as a rotating dipole imply a precise molecular design. In this respect, the hybrid organo-siloxane bricks are very versatile, because they can be synthesized with a variety of substituted aromatic groups and selfassemble into an organized mesoporous crystal, as depicted above. In order to design dipolar rotors, molecular dipole moment must not align with the rotation axis, and dipoles should be generated by low bulkiness atoms. Thus, the appropriate substitution of one or two hydrogen atoms of a pphenylene ring with a fluorine atom, generated dipoles that can rotate even if the inertial mass and the lateral encumbrance would be slightly larger.

These achievements have been recently accomplished in fluorinated mesoporous organosilicas (Figure 11). Indeed, two new compounds divinyl-monofluoro- and difluoro-pphenylenesilicas, denominated FOS-1 and FOS-2, were prepared and showed high surface areas and excellent adsorption behavior towards carbon dioxide. ${ }^{48}$ Dipole dynamics was studied by dielectric relaxation measurements that highlighted an intense dipole response at variable frequency and temperature. The maximum dielectric losses become evident when the applied electric frequency matches the molecular dipole dynamics. Under these conditions, dielectric response, typical of a fast dipole reorientation, is obtained by the stimuli of an applied electric field.

Furthermore, the mesochannels are open and accessible to gas molecule diffusion, and rotor-mobility could be individually regulated by $\mathrm{I}_{2}$ vapors. The iodine enters the channels of the periodic structure and reacts with the pivotal double bonds of the divinylfluoro-phenylene rotors, affecting their motion and the dielectric properties. This constitutes an intriguing example of solid state reaction affecting massively both the molecular dynamics and the macroscopic properties of a material.
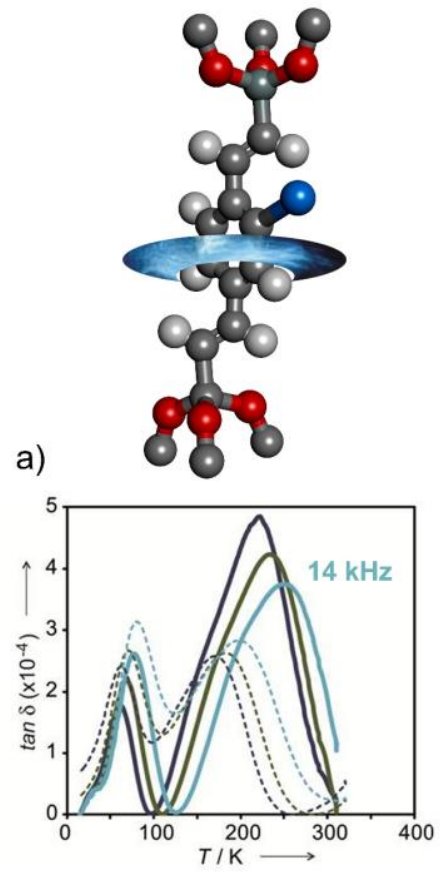

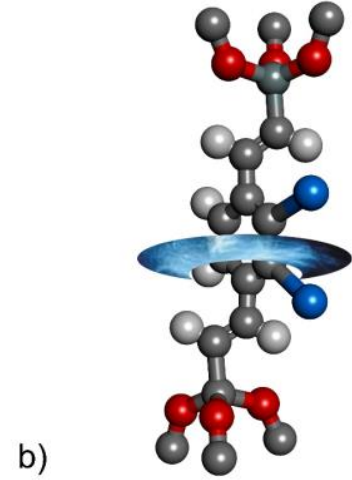

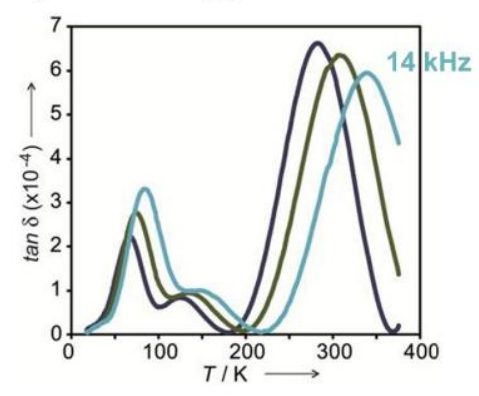

Figure 11. Dielectric loss vs temperature of FOS-1 (a) and FOS-2 (b) at 140, 1400 and $14000 \mathrm{~Hz}$.

\section{PERSPECTIVES}

Molecular motion in solid materials is a fascinating phenomenon, especially if a quite rigid and robust framework coexists with dynamics. Here, we discussed our contribution and that from other groups regarding this aspect, in conjunction with the feature of some solids of being porous and accessible from the gas phase. An additional step forward has been achieved when dielectric properties and dipole dynamics were realized. By this way, a few prerogatives were put together in an unconventional manner. Pioneering work demonstrates the feasibility of rotor-dynamics control by the action of chemical species absorbed from the vapor or gas phase, which intervene directly on the rotor dynamics, with the challenging perspective of building selective sensors and devices for release of chemicals on command. Moreover, the robustness of the covalent frameworks cohabits, paradoxically, with the motional behavior of the rotating elements and considerably extends the working temperature range.

\section{AUTHOR INFORMATION}

Piero Sozzani

* piero.sozzani@mater.unimib.it

Biographies

Piero Sozzani is full professor of Macromolecular Chemistry. He has been Visiting Scientist and consultant at At\&T Bell Laboratories. His main research interests encompass the study of nanostructured materials, polymerization in confined spaces and solid state NMR spectroscopy.

Angiolina Comotti received her PhD in Chemistry at the University of Milan and is currently Associate Professor. She was Visiting Scientist at ETHZ and NYU. Her main activity is 
focused on supramolecular chemistry and porous materials with particular interests on structural and dynamical aspects.

Silvia Bracco received her Phd in Materials Science. She was a post-doctoral fellow at UCSB and is currently Assistant Professor. Her activity deals with porous materials and solid state NMR.

\section{Author Contributions}

The manuscript was written through contributions of P.S., A.C. and S.B. authors. All authors have given approval to the final version of the manuscript.

\section{Funding Sources}

PRIN-MIUR and Cariplo Foundation are acknowledged for financial supports.

\section{REFERENCES}

(1) Kottas, G.S.; Clarke, L.I.; Horinek, D.; Michl, J. Artificial Molecular Rotors. Chem.Rev. 2005105,1281-1376.

(2) Vogelsberg, C.S.; Garcia-Garibay, M.A. Crystalline Molecular Machines: Function, Phase Order, Dimensionality, and Composition. Chem.Rev.Soc. 2012,41,1892-1910.

(3) Sozzani, P.; Bracco, S.; Comotti, A.; Simonutti, R. Motional Phase Disorder of Polymer Chains as Crystallized to Hexagonal Lattices. Adv.Polym.Sci. 2005,181,153-177.

(4) Molecular Machines and Motors; Credi, A., Silvi, S., Venturi, M., Eds.; Springer: Heidelberg, Germany, 2014.

(5) Browne, W.R.; Feringa, B.L. Making Molecular Machines Work. Nature Nanotech. 2006,1,25-35.

(6) Crowley, J.D.; Kay, E.R.; Leigh, D.A. In Intelligent Materials; Shahinpoor, M., Schneider, H.-J., Eds.; Royal Society of Chemistry: Cambridge, UK, 2008; pp1-47.

(7) Rodriguez-Velamazan, J.A.; Gonzalez, M. A.; Real, J.A.; Castro, M.; Munoz, M.C.; Gaspar, A.B.; Ohtani, R.; Ohba, M.; Yoneda, K.; Hijikata, Y.; Yanai, N.; Mizuno, M.; Ando, H.; Kitagawa, S. A Switchable Molecular Rotator: Neutron Spectroscopy Study on a Polymeric Spin-Crossover Compound. J.Am.Chem.Soc. 2012,134,5083-5089.

(8) Zhang, Y.; Zhang, W.; Li, S.H.; Ye, Q.; Cai, H.L.; Deng, F.; Xiong, R.G.; Huang, S.D. Ferroelectricity Induced by Ordering of Twisting Motion in a Molecular Rotor. J.Am.Chem.Soc. 2012,134,1104411049.

(9) Lemouchi, C.; Mézière, C.; Zorina, L.; Simonov, S.; RodriguezFortea, A.; Canadell, E.; Wzietek, P.; Auban-Senzier, P.; Pasquier, C.; Giamarchi, T.; Garcia-Garibay, M.A.; Batail, P. Design and Evaluation of a Crystalline Hybrid of Molecular Conductors and Molecular Rotors. J.Am.Chem.Soc. 2012,134,7880-7891.

(10) Setaka, W.; Yamaguchi, K. Order-Disorder Transition of Dipolar Rotor in a Crystalline Molecular Gyrotop and Its Optical Change. J.Am.Chem.Soc. 2013,135,14560-14563.

(11) NMR of Polymers; Bovey, F.A.; Mirau, P., Eds.; Academic Press: San Diego, USA, 1996.

(12) Bracco, S.; Comotti, A.; Simonutti, R.; Camurati, I.; Sozzani, P. Low-Temperature Crystallization of Ethylene-ran-propylene Copolymers: Conformational Rearrangement of Sequences During the Formation of the Aggregates. Macromolecules 2002,35,16771684 .

(13) Zhou, H.C.; Long, J. R.; Yaghi, O. M. Introdution to Metal-Organic Frameworks. Chem.Rev. 2012,112,673-674 and articles therein.

(14) Holst, J.R.; Trewin, A.; Cooper, A. I. Porous Organic Molecules. Nature Chem. 2010,2,915-920.

(15) Ben, T.; Shilun, Q. Porous Aromatic Frameworks: Synthesis, Structure and Functions. Cryst.Eng.Comm. 2013,15,17-26.

(16) Fujita, S.; Inagaki, S. Self-Organization of Organosilica Solids with Molecular-Scale and Mesoscale Periodicities. Chem.Mater. 2008,20,891-908.
(17) Comotti, A.; Valsesia, P.; Chmelka, B.F.; Sozzani, P. Molecular Rotors in Hierarchically-Ordered Mesoporous Silica Frameworks. Chem.Commun. 2008,4798-4800.

(18) Kubo, R.; Tomita, K. A General Theory of Magnetic Resonance Absorption. J.Phys.Soc.Jpn. 1954,9,888-919.

(19) Hoatson, G.L.; Vold, R.L. In 2H-NMR Spectroscopy of Solids and Liquid Crystals; NMR Basic Principles Prog. Bluemich, B. Ed.; Springer-Verlag 1994,32,1-67.

(20) Comotti, A.; Bracco, S.; Valsesia, P.; Beretta, M.; Sozzani, P. Fast Molecular Rotor Dynamics Modulated by Guest Inclusion in a Highly Organized Nanoporous Organosilica. Angew.Chem.Int.Ed. 2010,49,1760-1764.

(21) The energy value was established after the re-evaluation of temperature range.

(22) Comotti, A.; Bracco, S.; Valsesia, P., Ferretti, L.; Sozzani, P. 2D Multinuclear NMR, Hyperpolarized Xenon, and Gas Storage in Organosilica Nanochannels with Crystalline Order in the Walls. J.Am.Chem.Soc. 2007,129,8566-8576.

(23) Vogelsberg, S.C.; Bracco, S.; Beretta, M.; Comotti, A.; Sozzani, P.; Garcia-Garibay, M.A. Dynamics of Molecular Rotors Confined in Two Dimensions: Transition from a 2D Rotational Glass to a 2D Rotational Fluid in a Periodic Mesoporous Organosilica. J.Phys.Chem.B 2012,116,1623-1632.

(24) Horike, S.; Matsuda, R.; Tanaka, D.; Matsubara, S.; Mizuno, M.; Endo, K.; Kitagawa, S. Dynamic Motion of Building Blocks in Porous Coordination Polymers. Angew.Chem.Int.Ed. 2006,45,72267230

(25) Kolokolov, D.I.; Jobic, H.; Stepanov, A.G.; Guillerm, V.; Devic T.; Serre, C.; Ferey, G. Dynamics of Benzene Rings in MIL-53(Cr) and MIL-47(V) Frameworks Studied by ${ }^{2} \mathrm{H}$ NMR Spectroscopy. Angew.Chem.Int.Ed. 2010,49,4791-4794.

(26) Devautour-Vinot, S.; Maurin, G.; Serre, C.; Horcajada, P.; Paula da Cunha, D.; Guillerm, V.; de Souza Costa, E.; Taulelle, F.; Martineau, C. Structure and Dynamics of the Functionalized MOF Type Uio-66(Zr): NMR and Dielectric Relaxation Spectroscopies Coupled with DFT Calculations. Chem.Mater. 2012,24,2168-2177.

(27) Winston, E.B.; Lowell, P.J.; Vacek, J.; Chocholousova, J.; Michl, J.; Price, J.C. Dipolar Molecular Rotors in the Metal-Organic Framework Crystal IRMOF-2. Phys.Chem.Chem.Phys. 2008,10,5188-5191.

(28) Morris, W.; Taylor, R.E.; Dybowski, C.; Yaghi, O.M.; GarciaGaribay, M.A. Framework Mobility in the Metal-Organic Framework Crystal IRMOF-3: Evidence for Aromatic Ring and Amine Rotation. J.Mol.Struct. 2011,1004,94-101.

(29) Sozzani, P.; Bracco, S.; Comotti, A.; Ferretti, L. Simonutti, R. Methane and Carbon Dioxide Storage in a Porous Van der Waals Crystal. Angew.Chem.Int.Ed. 2005,44,1816-1820.

(30) Yadav, V.N.; Comotti, A.; Sozzani, P.; Bracco, S.; Bonge-Hansen, T.; Hennum, M.; Gorbitz, C.H. Microporous Molecular Materials from Dipeptides Containing Non-proteinogenic Residues. Angew.Chem.Int.Ed. 2015,54,684-15688.

(31) Mastalerz, M. Permanent Porous Materials from Discrete Organic Molecules-Towards Ultra-High Surface Areas. Chem.Eur.J. 2012,18,10082-10091.

(32) Baroncini, M.; d'Agostino, S.; Bergamini, G.; Ceroni, P.; Comotti, A.; Sozzani, P.; Bassanetti, I.; Grepioni, F.; Hernandez, T. M.; Silvi, S.; Venturi, M.; Credi, A. Photoinduced Reversible Switching of Porosity in Molecular Crystals Based on Star-Shaped Azobenzene Tetramers. Nature Chem. 2015, 7, 634-640.

(33) Sozzani, P.; Comotti, A.; Simonutti, R.; Meersmann, T.; Logan, J.W.; Pines, A. A Porous Crystalline Molecular Solid Explored by Hyperpolarized Xenon. Angew.Chem.Int.Ed. 2000,39,26952698.

(34) Comotti, A.; Bracco, S.; Ferretti, L.; Mauri, M.; Simonutti, R.; Sozzani, P. A Single-Crystal Imprints Macroscopic Orientation to Xenon Atoms. Chem.Commun. 2007,350-352.

(35) Comotti, A.; Bracco, S.; Yamamoto, A.; Beretta, M.; Hirukawa, T.; Tohnai, N.; Miyata, M.; Sozzani, P. Engineering Switchable Rotors in Molecular Crystals with Open Porosity. J.Am.Chem.Soc. 2014,136,618-621. 
(36) Schilling, F.C.; Amundson, K.R.; Sozzani, P. Molecular Motion of Isolated Polyethylene Chains in the Solid State. Macromolecules 1994,27, 6498-6502.

(37) Schilling, F.C.; Sozzani, P.; Bovey, F. Chain Conformation and Dynamics of Crystalline 1,4-trans-Polyisoprene and its Inclusion Compound with Perhydrotriphenylene. Macromolecules 1991,24,4369-4375.

(38) Sozzani, P.; Behling, R.W.; Schilling, F.C.; Bruckner, S.; Helfand, E.; Bovey, F.A.; Jelinsky, L.W. Traveling Defects in 1,4Trans-Polybutadiene as an Inclusion Complex in Perhydrotriphenylene Canals and a Comparison with Molecular Motions in the Crystalline Solid-State. Macromolecules 1989, 22, 3318-3322.

(39) Harris, K.D.M. In Fundamental and Applied Aspects of Urea and Thiourea Inclusion Compounds; Supramolecular Chemistry; Taylor \& Francis; 2007,19,47-53.

(40) Comotti, A.; Simonutti, R.; Catel, G.; Sozzani, P. Isolated Linear Alkanes in Aromatic Nanochannels. Chem. Mater. 1999, 11, 1476-1483.

(41) Sozzani, P.; Comotti, A.; Bracco, S.; Simonutti, R. Cooperation of Multiple $\mathrm{CH}$...? Interactions to Stabilize Polymers in Aromatic Nanochannels as Indicated by 2D Solid State NMR. Chem.Comm. 2004,768-769.

(42) Becker, J.; Comotti, A.; Simonutti, R.; Sozzani. P.; Saalwachter, K. Molecular Motion of Isolated Linear Alkanes in Nanochannels. J.Phys.Chem.B 2005,109,23285-23294.

(43) Sozzani, P.; Comotti, A.; Bracco, S.; Simonutti, R. A Family of Supramolecular Frameworks of Polyconjugated Molecules Hosted in Aromatic Nanochannels. Angew.Chem.Int.Ed. 2004,43,27922797.

(44) Brustolon, M.; Barbon, A.; Bortolus, M., Maniero, A.L.; Sozzani, P.; Comotti, A.; Simonutti, R. Dynamics of alkoxy-oligothiophene ground and excited states in nanochannels. J.Am.Chem.Soc. 2004,126,15512-15519.

(45) Kobr, L.; Zhao, K.; Shen, Y.Q. ; Comotti, A.; Bracco, S.; Shoemaker, R.K.; Sozzani, P.; Clark, N.A.; Price, J.C.; Rogers, C. T.; Michl, J. Inclusion Compound Based Approach to Arrays of Artificial Dipolar Molecular Rotors. A Surface Inclusion. J.Am.Chem.Soc. 2012,134,10122-10131.

(46) Kobr, L.; Zhao, K.; Shen, Y.Q.; Polivkova, K.; Shoemaker, R.K.; Clark, N.A.; Price, J.C., Rogers, C.T.; Michl, J. Inclusion Compound Based Approach to Arrays of Artificial Dipolar Molecular Rotors: Bulk Inclusions. J.Org.Chem. 2013,78,1768-1777.

(47) Comotti, A.; Bracco, S.; Ben, T.; Qiu, S.; Sozzani , P. Molecular Rotors in Porous Aromatic Frameworks. Angew.Chem.Int. Ed. 2014,53,1043-1047.

(48) Bracco, S.; Beretta, M.; Cattaneo, A.; Comotti, A.; Falqui, A.; Zhao, K.; Rogers, C.; Sozzani, P. Dipolar Rotors Orderly Aligned in Mesoporous Fluoro-Organosilica Architectures. Angew.Chem.Int.Ed. 2015,54,4773-4777. 